Journal of Animal and Veterinary Advances 11 (9): 1498-1511, 2012

ISSN: $1680-5593$

(C) Medwell Journals, 2012

\title{
The Diversity of Plant Species in Rangelands (Ordu Region, Turkey)
}

\author{
Metin Deveci \\ Department of Field Crops, Faculty of Agriculture, Ordu University, 52200 Ordu, Turkey
}

\begin{abstract}
The study was carried out in the rangelands of Ordu located in the Euro-Siberian floral region between 2009-2010 is to present the plant species diversity of the local rangelands. In this study, 246 genera, 461 species and subspecies belongs to 61 different families were identified in the research area. The distribution of taxa according to phytogeographic regions is as follows: $42.28 \%$ Euro-Siberian, 5.94\% Irano-Turanian, 4.04\% Mediterranean and $47.74 \%$ unknown region or widely distributed. The families with the largest number of genera are Asteraceae $(48 ; 11.85 \%)$, Fabaceae $(47 ; 11.16 \%)$ and Poaceae $(43 ; 10.21 \%)$, respectively. The richest genera are Trifolium L. (16 taxa), Campanula L. (10 taxa) and Medicago L. (8 taxa). The distribution of taxa according to life-form is as follows: 261 Hemicryptophyte $(62.00 \%), 83$ Therophyte (19.72\%), 40 Geophyte $(7.96 \%), 23$ Chamaephyte $(5.46 \%), 8$ Phanerophyte (1.90\%) and 6 Hydrophyte (1.43\%). The study identified 421 taxa of which 318 are perennial, 76 are annual, 19 are biennial, 3 are annual and biennial and 5 are biennial and perennial. The endemism rate is $6.89 \%$ ( 29 taxa). The risk status of 15 taxa was evaluated according to International Union for Conservation of Nature and Natural Resources (IUCN) risk criteria.
\end{abstract}

Key words: Plant species, endemic plants, rangelands, Euro-Siberian, Ordu, Turkey

\section{INTRODUCTION}

Biological diversity is the most important natural richness of a country. The conservation and maintenance of national biological diversity are of great importance for sustainable global biological diversity and natural balance. Turkey is one of the most important centers in the world with relations to the flora. The floristically rich region is the gene center of many plants found around the world.

Erik and Tarikahya (2004) reported that almost 12,000 different plant taxa were found within Turkey. With the latest new genera and records by Ozhatay and Kultur (2006) and Ozhatay et al. (2009) this number reached 12.476. The endemism ratio is approximately $34 \%$ with 3925 endemic species (NPNC, 2007). There are three different flora regions represented in Turkey, EuroSiberian, Mediterranean and Irano-Turanian (Davis, 1985). The Euro-Siberian floral region is divided into the Euxin and Hyrcanien. The Black sea region in Euxin takes the fourth place with 220 species in relation with the number of species grown in a single region within the country (Ekim et al., 2000).

Many studies have been carried out in order to determine the flora of the Black sea region, a great majority of which includes the Western and Eastern Black sea region. Ansin (1980) identified 2239 different plant species and determined 222 endemic plant taxa in the Eastern Black sea Region. Further studies were carried out in the same region by Ansin (1983), Karakaya and Kilinc
(1996), Eminagaoglu and Ansin (2003, 2004), Palaba and Ansin (2006), Uzun and Terzioglu (2008), Severoglu at al. (2011) and Deveci (2012). In addition to species richness, diversity existed within the species. Asci (2011a, b) reproted that large variation existed among the red clover genotypes, collected from Black sea region in terms of morpho-agronomic properties and salt tolerance.

Rangelands is one of the most important among natural resources of the earth that produce a wide variety of goods and services desired by society, including livestock forage, wildlife habitat, water, mineral resources, wood products, wildland recreation, open space and natural beauty. Rangelands are rich in biological diversity are also the gene source of plant and animal organisms. Soil is protected through the vegetation on rangelands also rangelands reduced the greenhouse effect and warming of the earth. In addition, the formation of water resources are also important for the development and preservation.

The rangelands of Ordu region as the field study are located in the zone Euro-Siberian floristic regions. In this regard, the aim of this study is to determine the plant species diversity of the endemic and endangered plant species which have the feature of a biological heritage for the country.

\section{MATERIALS AND METHODS}

The material of this study, carried out during 2009-2010 is comprised of the vegetation cover in the zone of Ordu city. According to development periods of plants, 


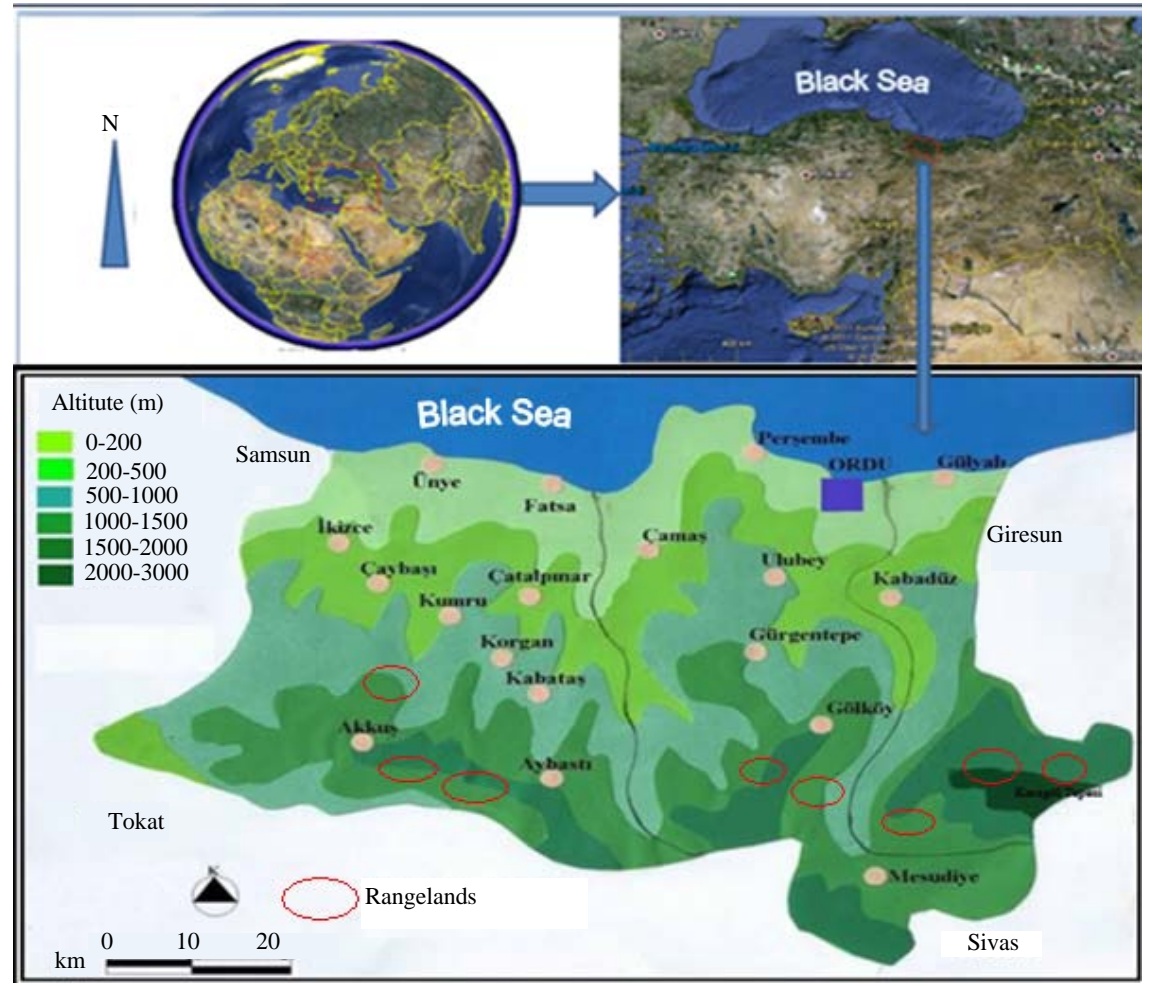

Fig. 1: Ordu Region and its location in Turkey

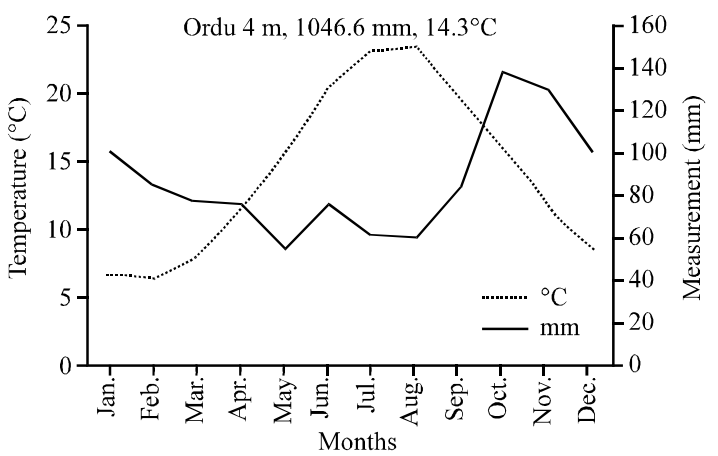

Fig. 2: Climatic diagram of province in Ordu region

attempts were made to identify the plants in the field, especially during their generative periods; studies were conducted along the line of Mesudiye town and Cambasi high plateau rangelands $(1650 \mathrm{~m})$, Golkoy Aydogan Alanyurt plateau rangelands $(1450 \mathrm{~m})$, Persembe plateau rangeland $(1550 \mathrm{~m})$, Kumru Duzoba plateau rangelans (1475 m), Kabaduz Sinanli-Hummetli Pleteau Rangelands $(1460 \mathrm{~m}$ ) situated within the province of Ordu (Fig. 1).

Regional climatic data for 1975-2010 (35 years) shows that Ordu generally experience drought during July and August (Fig. 2). Frost can be seen between the second half of September and April on the coast of Ordu. According to the long-term data, the highest annual precipitation of Ordu was $1046.6 \mathrm{~mm}$. According to the long term data, annual average temperature was $14.3^{\circ} \mathrm{C}$. The average temperature values of the hottest month $(\mathrm{M})$ was August $\left(27.7^{\circ} \mathrm{C}\right)$ and the coldest month $(\mathrm{m})$ was February $\left(3.6^{\circ} \mathrm{C}\right)$ in Ordu. The hottest and coldest temperature in Ordu was determined to be during June $\left(37.3^{\circ} \mathrm{C}\right)$ and February $\left(-6.7^{\circ} \mathrm{C}\right)$. The relative humidity level is much higher during summer than during winter. The prevailing wind direction is South-Southeast in Ordu. A climate diagram of the cities within the research area was prepared using the Walter Method (Akman, 1999). Ordu region generally has a mild oceanic climate in which precipitation is evenly distributed throughout the year (Akman, 1999).

The forest flora of the region is comprised of various trees, shrubs and herbaceous and woody living covers. Moreover, meadows, pastures and high plateaus embody important plant diversity. In terms of agricultural products, tea and hazelnut are peculiar to the region and a large variety of additional products are grown including land, vegetable and fruit groups.

The study was conducted during the vegetation period between April and Novomber in both years and asseement of the flora was performed during these periods by visiting the rangelands which each had different topographical structures semimonthly. 
Samples were taken of any unidentified plants which were subsequently identified according to Davis (1985) by preparing herbarium samples. Furthermore, the digital photographs of plant species obtained from the areas were taken. The rangelands where the plants were obtained and their picking times were noted. The plants came into blossom at different times therefore, obtaining samples from the plants with different blossom periods were ensured by visiting the same lands at various dates in the vegetation period.

The plants obtained from the research area were classified alphabetically according to their class, family, genus and species. The classification list included the lifespans of the plants (Anonymous, 2008), habitus types, life forms (Raunkiaer, 1934; Ellenberg and Mueller-Dombois, 1967; Andic, 1977, 1985; Yaltirak and Efe, 1996; Deveci and Andic, 1992), their floristic regions and whether or not they are endemic (Davis, 1985; Baytop, 1997). The risk categories of the endemic and non-endemic taxa identified in the region were determined according to IUCN criteria (Ekim et al., 2000; IUCN, 2001).

\section{RESULTS AND DISCUSSION}

A total of 421 taxa were determined at the level of species, subspecies and variety which belong to 246 genera of 61 families out of 1500 plant samples taken from the region during the research.

Given the distribution of the plants in the research area according to the floristic regions, 201 taxa (47.74\%) were classed as having more than one region or as having no specific region. The Euro-Siberian (178; 42.28\%) element was found to be most widespread followed respectively by the Irano-Turanian $(25 ; 5.94 \%)$ and Mediterranean (17; 4.04\%) elements (Table 1). The high inclusion rate of Euro-Siberian element indicates that the region is a part of this floral element. Similar values in terms of each three floral elements were reported in previous studies in the same and nearby regions (Deveci, 2012; Karakaya and Kilinc, 1996; Eminagaoglu and Ansin, 2003, 2004; Palaba and Ansin, 2006; Uzun and Terzioglu, 2008) (Table 2). The Euro-Siberian elements seem to be dominant in all areas studied while the Irano-Turanian elements come second, Mediterranean elements third in all these areas.

Twenty nine of the determined taxa are endemic $(6.89 \%)$. Endemic taxa are listed as Euro-Siberian (18; $4.28 \%)$, Irano-Turanian $(5 ; 1.19 \%)$ and unknown or with more than one origin $(6 ; 1.43 \%)$ (Table 1$)$. The results for endemism were same compared to previous studies carried out in similar regions (Karakaya and Kilinc, 1996; Eminagaoglu and Ansin, 2003, 2004; Palaba and Ansin, 2006; Uzun and Terzioglu, 2008) (Table 2). This rate is low
Table 1: Phytogeographic, endemic, nonendemic and threat categaries distribution of the plant taxa located in the study area

\begin{tabular}{|c|c|c|c|c|c|c|}
\hline \multirow{3}{*}{$\begin{array}{l}\text { Phytogeographical } \\
\text { region }\end{array}$} & \multirow{2}{*}{\multicolumn{2}{|c|}{ Endemic }} & \multicolumn{2}{|c|}{ Nonendemic } & \multicolumn{2}{|c|}{ Total } \\
\hline & & & $\cdots$ & --------י & $-\cdots$ & ---- \\
\hline & No. & $\%$ & No. & $\%$ & No. & $\%$ \\
\hline Euro-Sib. & 18 & 4.28 & 160 & 38.00 & 178 & 42.28 \\
\hline Ir.-Tur. & 5 & 1.19 & 20 & 4. 75 & 25 & 5.94 \\
\hline Medit. & - & - & 17 & 4.04 & 17 & 4.04 \\
\hline Mul. or Unk. P.Reg. & 6 & 1.43 & 195 & 46.78 & 201 & 47.74 \\
\hline Total & 29 & 6.89 & 392 & - & 421 & 100.00 \\
\hline CR & - & - & 1 & 0.24 & 1 & 0.24 \\
\hline $\mathrm{EN}$ & 2 & 0.48 & - & - & 2 & 0.48 \\
\hline vU & 2 & 0.48 & 1 & 0.24 & 3 & 0.71 \\
\hline LR (nt) & 2 & 0.48 & - & - & 2 & 0.48 \\
\hline LR (lc) & 8 & 2.14 & 1 & 0.24 & 10 & 2.36 \\
\hline Total & 14 & - & 3 & - & 18 & - \\
\hline
\end{tabular}

Table 2: Comparison (\%) of floristic results between the present study and other studies conducted in adjacent or nearby areas according to number of taxa, the phytogeographical elements and endemism

\begin{tabular}{lccccc}
\hline & $\begin{array}{c}\text { No. } \\
\text { of taxa }\end{array}$ & $\begin{array}{c}\text { Euro- } \\
\text { Sib. }\end{array}$ & $\begin{array}{c}\text { Ir.- } \\
\text { Tur. }\end{array}$ & Medit. & Endemism \\
\hline Present study & 421 & 42.28 & 5.94 & 4.04 & 6.9 \\
Deveci (2012) & 540 & 40.56 & 7.78 & 2.96 & 11.5 \\
Uzun and Terzioglu (2008) & 383 & 48.04 & 3.66 & 2.87 & 4.2 \\
Palaba and Ansin & 384 & 50.27 & 5.99 & 1.04 & 8.3 \\
$\begin{array}{l}\text { (2006) } \\
\text { Eminagaoglu and Ansin }\end{array}$ & 872 & 39.40 & 10.30 & 1.10 & 6.3 \\
(2004) & & & & & \\
Eminagaoglu and Ansin (2003) & 769 & 35.60 & 6.90 & 2.20 & 7.4 \\
Karakaya and Kilinc (1996) & 323 & 46.74 & 4.03 & 0.93 & 8.7 \\
\hline
\end{tabular}

compared with the averange endemism rate of the flora of Turkey (34.5\%) (Guner et al., 2000) because the region is lower than that of the other two phytogeographical region. Moreover, Ansin et al. (2002) reported that the rate of endemism in a study carried out in the Eastern Black sea region was almost $16 \%$. However, a study conducted in the same region Zone Colchic were found higher rate of endemism (11.6\%) (Deveci, 2012). The rate of endemism were lower found in this study because this study was only carried in rangeland areas of the region. Babalik and Fakir (2010) reported that the endemism rate in Isparta region rangelans was $27.15 \%$. Because Isparta located in Mediterranean region takes the first place with 750 taxa in relation with the number of species grown in a single region within the Turkey (Ekim et al., 2000).

A total of 14 taxa, all endemic and 3 nonendemic taxa were evaluated according to IUCN risk categories (Ekim et al., 2000; IUCN, 2001). The distribution of the threat categories is as follows: 1 nonendemic taxa in CR, 2 endemic taxa in EN, 2 endemic and 1 nonendemic taxa in VU, 2 endemic taxa in LR (nt), 9 endemic and 1 nonendemic taxa in LR (lc) (Table 1).

The richest family in terms of number of taxa was Asteraceae (Compositae) (48; 11.40\%). Additionaly, it is followed by Fabaceae (Leguminosae) (47; 11.16\%), Poaceae (Gramineae) (43; 10.21\%), Lamiaceae (Labiatae) $(28 ; 6.65 \%)$, Scrophulariaceae and Rosaceae $(23 ; 5.46 \%)$, Apiaceae (Umbelliferae) $(16 ; 3.80 \%)$, Liliaceae $(14 ; 3.33 \%)$, 
Campanulaceae $(12 ; 2.85 \%)$ and Brassicaceae (Cruciferae) $(8 ; 1.90 \%$ ) (Table 3 and 4 ). The results are smilar to those of Babalik and Fakir (2010) in respect to floristic composition. However, the results of this study are higher than those of other similar studies (Eminagaoglu and Ansin, 2004; Palaba and Ansin, 2006; Uzun and Terzioglu, 2008; Deveci, 2012). Differences in diversity of plant might be due to the results of dissimilarities in climates and habitats.

Asteraceae (Compositae) (the largest family in the list) is one of the largest family in the Flora of Turkey (Guner et al., 2000). The Asteracea, Fabaceae and Poaceae families are the richest families in terms of nummer of taxa and these results are very similar to those in the study (Table 3).

The richest genera in terms of the number of taxa in study area were Trifolium L. (16), Campanula L. (10), Geranium L. and Medicago L. (8) and Veronica L. (6) (Table 5).

The Fabaceae family varies in habit from annual and perennial herbs to shrubs, trees and even a few aquatics and therefore it is cosmopolitan in distribution and wellrepresented throughout temperate and tropical regions of the world (Rundel, 1989). The preference of Fabaceae members for semi-arid to arid habitats is related to a

Table 3: Numerical and dispersion rates of 10 families containing the most taxa identified in the study area

\begin{tabular}{lcc}
\hline Family & No. of taxa & Rates (\%) \\
\hline Asteraceae & 48 & 11.40 \\
Fabaceae & 47 & 11.16 \\
Poaceae & 43 & 10.21 \\
Lamiaceae & 28 & 6.65 \\
Scrophulariaceae & 23 & 5.46 \\
Rosaceae & 23 & 5.46 \\
Apiaceae & 16 & 3.80 \\
Liliaceae & 14 & 3.33 \\
Orchidaceae & 13 & 3.09 \\
Campanulaceae & 12 & 2.85 \\
Brassicaceae & 8 & 1.90 \\
Other families & 146 & 34.68 \\
Total & 421 & 100.00 \\
\hline
\end{tabular}

nitrogen-demanding metabolism which is thought to be an adaptation to climatically variable or unpredictable habitats (McKey, 1994). Turkey is the richest Mediterranean country for Trifolium genus with over 100 species in its natural flora (Zohary and Heller, 1984). The most common genera were Trifolium (16 taxa), Medicago (8 taxa), Lathyrus (5 taxa) and Vicia (4 taxa). Trifolium and Medicago genera are listed in all provious researches realized in the Eastern Black sea region of Turkey (Davis, 1985; Ansin, 1980, 1983; Eminagaoglu and Ansin, 2003, 2004; Uzun and Terzioglu, 2008; Severoglu et al., 2011).

The life-form spectrum of the taxa in this study was as follows: Hemicryptophytes $261(62.00 \%)$, Therophytes $83(19.72 \%)$, Geophytes $40(9.50 \%)$, Chamaephytes 23 $(5.46 \%)$, Phanerophytes $8(1.90 \%)$ and Hydrophytes 6 $(1.43 \%)$. Hemicryptophytes were the best represented class in the study area (Fig. 3). The results of this study are in agreement with those of other similar studies (Altay et al., 2010; Severoglu et al., 2011). Hemicryptophytes have been followed by therophytes; annual plants which survive the unfavorable season in the form of seeds and complete their life-cycle during favorable seasons. The 20 taxa of Hemicryptophytes are endemic.

About 318 taxa among 421 plants taxa are perrennial, 76 of the plants are annual, 19 of the plants are biannual, 3 of them are annual and biannual plants and 5 of them are biannual and perennial plants (Table 4).

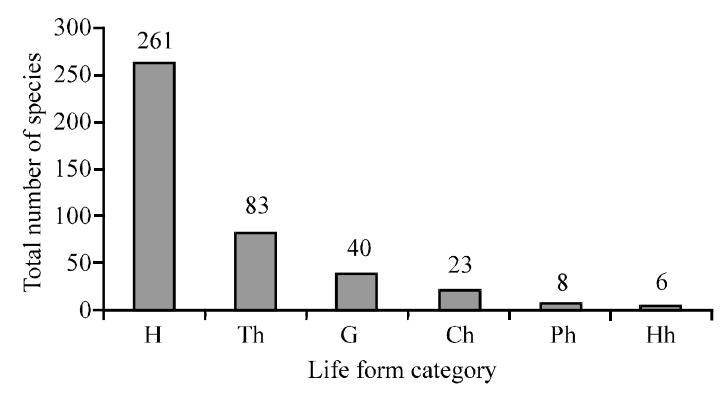

Fig. 3: Life form spectrum of taxa

Table 4: Comparison of 10 families containing the most species in studies conducted in nearby regions (\%)

\begin{tabular}{|c|c|c|c|c|c|c|}
\hline Family & Present study & Deveci (2012) & $\begin{array}{l}\text { Babalik and } \\
\text { Fakir }(2010)\end{array}$ & $\begin{array}{l}\text { Uzun and Terzioglu } \\
(2008)\end{array}$ & $\begin{array}{l}\text { Palaba and Ansin } \\
(2006)\end{array}$ & $\begin{array}{c}\text { Eminagaoglu and } \\
\text { Ansin (2004) }\end{array}$ \\
\hline Asteraceae & 11.4 & 11.9 & 13.6 & 9.1 & 10.2 & 10.3 \\
\hline Fabaceae & 11.2 & 10.0 & 12.9 & 6.0 & 6.0 & 8.8 \\
\hline Poaceae & 10.2 & 8.7 & 9.6 & 2.6 & 7.6 & 6.9 \\
\hline Lamiaceae & 6.7 & 6.1 & 9.9 & 7.0 & 3.1 & 5.7 \\
\hline Scrophulariaceae & 5.5 & 4.3 & 3.3 & 5.0 & 6.3 & 5.1 \\
\hline Rosaceae & 5.5 & 4.1 & 2.0 & 5.7 & 9.1 & 6.4 \\
\hline Apiaceae & 3.8 & 3.2 & 2.0 & 4.4 & 2.3 & 2.2 \\
\hline Liliaceae & 3.3 & 3.0 & 2.7 & 2.9 & 3.9 & 2.8 \\
\hline Brassicaceae & 1.9 & 2.6 & 8.0 & 4.7 & 2.3 & 5.2 \\
\hline Campanulaceae & 2.9 & 2.6 & 2.3 & 1.8 & 2.9 & 1.8 \\
\hline Total & 62.4 & 56.5 & 66.3 & 49.2 & 53.7 & 55.2 \\
\hline
\end{tabular}


Table 5: Length of life, life-form category, phytogeographic, endemic, nonendemic and threat categaries distribution of the plant taxa located in the study area

Family and taxa of plants

Pteridophyta

Aspleniaceae

Phyllitis scolopendrium (L.) Newm.

Aspidiaceae

Dryopteris affinis (Lowe) Fraser-Jenkins subsp. coriccea Fraser-Jenkins

Hypolepidaceae (Dennstaedtiaceae)

Pteridium aquilinum (L.) Kuhn.

Thelypteridaceae

Thelypteris limbosperma (All.) Fuchs.

Spermatophyta

Angiospermae

Dicotyledones

Anacardiaceae

Rhus coriaria L

Apiaceae (Umbellifer ae)

Ammi visnaga (L.) Lam.

Angelica sylvestris L. var. sylvestris $\mathrm{L}$

Anthriscus nemorosa (Bieb.) Sprengel.

Astrantia maxima Pallas subsp. maxima Pallas.

Carum meifolium (Bieb.) Boiss.

Caucalis platycarpos $\mathrm{L}$.

Chaerophyllum aureum $\mathrm{L}$.

Chaerophyllum byzontium Boiss.

Dancus carota L.

Eryngium giganteum Bieb.

Herccleum platytaenium Boiss.

Oenonthe pimpinelloides $\mathrm{L}$.

Pimpinella anthriscoides Boiss. var. cruciata (Bomm. Et Wolff.) Matthews.

Pimpinella rhodantha Boiss.

Torilis arvensis (Huds.) Link subsp. arvensis (Huds.) Link.

Turgenia latifolia (L.) Hoffm.

Araliaceae

Hedera colchica (C. Koch).

Asteraceae (Compositae)

Achillea millefolium $\mathrm{L}$. subsp. mille folium $\mathrm{L}$.

Anthemis tinctoria L.

Anthemis triumfettii (L.) All.

Arctium platylepis (Boiss.Et. Bal.) Sosn. Ex Grossh.

Aster alpinus L.

Aster cancasicus Willd.

Bellis perennis $\mathrm{L}$.

Carduss mutons $\mathrm{L}$

Carduus acanthoides L. subsp. aconthoides L.

Carlina vulgaris L.

Centaurea iberica Trev. Ex Sprengel.

Centaurea sessilis Willd.

Centaurea urvillei DC. subsp. urvillei DC.

Cicerbita racemosa (Willd.) Beauverd.

Cichorium intybus $\mathrm{L}$.

Cirsium arvense (L.) Scop. Subsp. arvense (L.) Scop.

Cirsium trachylepis Boiss.

Crepis sancta (L.) Babcock.

Erigeron acer L. subsp. acer L.

Erigeron caucasicus Stev. subsp. caucasic us Stev.

Eupatorium cannabinum L.

Gnophalium sylvaticum $\mathrm{L}$.

Helichrysum armenium DC. subsp. armenium DC.

Helichrysum plicatum DC. subsp. plicatum DC.

Hieracium erythrocarpum Peter.

Inula vulgaris (Lam.) Trevisan

Lapsana communis L. subsp. grandiflora (Bieb.)Sell.

Leontodon hispidus L. var. hispidus $\mathrm{L}$.

Leontodon tuberosus $\mathrm{L}$.

Matric aria chamomilla $\mathrm{L}$.

Mycelis muralis (L.) Dum.

Petasites hybridus (L.) Gaertner, Mey. Et Scherb.

Picnomon acarna (L.) Cass.

Picris hieracioides L.

Length of life Life-form

Element region End.

IUCN

Per.

Per.

$\mathrm{H}$

Per.

Per.

H

Euro-Sib.

H

$\mathrm{H}$

Per.

Bia

Per.

Per.

Per.

Per.

Ann.

Per.

Per.

Bia.

Bia.

Bia.

Per

Per.

Per.

Per.

Ann.

Per.

Per.

Per.

Per.

Bia.

Per.

Per.

Per.

Per.

Bia.

Bia

Per.

Per.

Per.

Per.

Per.

Ann.

Per.

Ann.

Per.

Per.

Per.

Per.

Per.

Per.

Per.

$\mathrm{Bia}$

Per.

Per.

Per.

Ann.

Per.

Per.

Ann.

Bia. Per.
Medit.

Euro-Sib.

Medit.

-

Euro-Sib.

Euro-Sib.

Euro-Sib.

Ir.-Tur.

-

Euro-Sib.

Euro-Sib.

Euro-Sib.

Euro-Sib.

Euro-Sib.

Euro-Sib.

Ir.-Tur.

Medit.

Euro-Sib.

-

Euro-Sib.

Euro-Sib.

Euro-Sib.

Euro-Sib.

Euro-Sib.

Ir.-Tur.

Euro-Sib.

Euro-Sib.

Euro-Sib.

Euro-Sib.

Medit

Euro-Sib.

Euro-Sib.

Medit.

Euro-Sib.
LR (lc)

LR (lc)

End. LR (lc)

- $\quad$ -

LR (nt)

- 
Table 5: Continue

\begin{tabular}{|c|c|c|c|c|c|}
\hline Family and taxa of plants & Length of life & Life-form & Element region & End. & IUCN \\
\hline Prenanthes cacaliifolia (Biebb.) Bbeauverd. & Per. & $\mathrm{H}$ & Euro-Sib. & - & - \\
\hline Ptilostemon afer (Jacq.) Greuter subsp. eburne us Greuter. & Bia. & $\mathrm{H}$ & - $\quad$ & End. & - \\
\hline Scorzonera cana (C.A. Meyer) Hoffm. var. cana (C.A. Meyer) Hoffm. & Per. & $\mathrm{H}$ & - & - & - \\
\hline Scorzonera pseudolanata Grossh. & Per. & $\mathrm{H}$ & Ir.-Tur. & - & - \\
\hline Senecio vulgaris $\mathrm{L}$. & Ann. & Th & - & - & - \\
\hline Solidago virgaurea $\mathrm{L}$. subsp. virgaure $\mathrm{L} \mathrm{L}$. & Per. & $\mathrm{H}$ & Euro-Sib. & - & - \\
\hline Tanacetum macrophyllum (Waldst. Et Kit.) Sscultz Bip. & Per. & $\mathrm{H}$ & Euro-Sib. & - & - \\
\hline Tanacetum parthenifolium (Willd.) Schultz Bip. & Per. & $\mathrm{H}$ & Ir.-Tur. & - & - \\
\hline Taraxacum crepidiforme DC. subsp. crepidiforme DC. & Per. & $\mathrm{H}$ & Ir.-Tur. & - & - \\
\hline Taraxacum turcicum Van Soest. & Per. & $\mathrm{H}$ & - & End. & - \\
\hline Telekia speciosa (Schreber) Baumg. & Per. & $\mathrm{H}$ & Euro-Sib. & - & - \\
\hline Tripleurospermum oreades (Boiss.) Rech. Fil. var. oreades (Boiss.) Rech. Fil. & Per. & $\mathrm{H}$ & - & - & \\
\hline Tussilago farfara $\mathrm{L}$. & Per. & $\mathrm{H}$ & Euro-Sib. & - & - \\
\hline Xeranthemum annusm $\mathrm{L}$. & Ann. & Th & - & - & - \\
\hline \multicolumn{6}{|l|}{ Balsaminaceae } \\
\hline \multicolumn{6}{|l|}{ Betulaceae } \\
\hline & Boraginaceae & $\mathrm{Ph}$ & Euro-Sib. & - & - \\
\hline Buglossoides arvensis (L.) Johnston. & Ann. & Th & - & - & - \\
\hline Cerinthe minor L. subsp. auriculata (Ten.) Domac. & Per. & $\mathrm{H}$ & - & - & - \\
\hline Echium vulgare L. & Bia. & Th & Euro-Sib. & - & - \\
\hline Myosotis alpestris F.W. Schmidt subsp. alpestris F.W. Schmidt. & Per. & $\mathrm{H}$ & - & - & - \\
\hline Myosotis lazica M. Podov. & Ann. Bia. & Th & Euro-Sib. & End. & vu \\
\hline Onosma sericeum Willd. & Per. & $\mathrm{H}$ & Ir.-Tur. & - & - \\
\hline Onosma tauricum Pallas Ex Willd. var. touricum Pallas Ex Willd. & Per. & $\mathrm{H}$ & - & - & - \\
\hline Symphytum asperum Lepechin var. asperum Lepechin. & Per. & $\mathrm{H}$ & Euro-Sib. & - & - \\
\hline Trachystemon orientalis (L.) G. Don. & Per. & $\mathrm{G}$ & Euro-Sib. & - & - \\
\hline \multicolumn{6}{|l|}{ Brassicaceae (Cruciferae) } \\
\hline Arabis caucasica Wild. subsp. caucasica Wild. & Per. & $\mathrm{H}$ & Medit. & - & - \\
\hline Capsella bursa-pastoris (L.) & Ann. & Th & - & - & - \\
\hline Cardamine bulbifera (L.) Crantz. & Per. & $\mathrm{G}$ & Euro-Sib. & - & - \\
\hline Cardamine lazica Boiss. E. Bal. & Per. & $\mathrm{G}$ & Euro-Sib. & - & - \\
\hline Cardomine quinquefolia (Bieb.) Schmalh. & Per. & Hh & Euro-Sib. & - & - \\
\hline Sincpis arvensis L. & Ann. & Th & - & - & - \\
\hline Rorippa sylvestre (L.) Bess. & Per. & $\mathrm{H}$ & - & - & - \\
\hline Thlaspi ochrolencum Boiss. Et Heldr. & Ann. & Th & - & - & - \\
\hline \multicolumn{6}{|l|}{ Campanulaceae } \\
\hline Asyneuma amplexicaule (Willd.) Hand.-Mazz. subsp. amplexicaule Willd. & Per. & $\mathrm{H}$ & - & - & - \\
\hline Companula alliariifolia Willd. & Per. & $\mathrm{H}$ & Euro-Sib. & - & - \\
\hline Campanula aucheri A. DC & Per. & $\mathrm{H}$ & Euro-Sib. & - & - \\
\hline Campanula bononiensis $\mathrm{L}$. & Per. & $\mathrm{H}$ & Euro-Sib. & - & - \\
\hline Campanula glomerata L. subsp. hispida (Witasek) Hayek. & Per. & $\mathrm{H}$ & Euro-Sib. & - & - \\
\hline Companula lactiflora Bieb. & Per. & $\mathrm{H}$ & Euro-Sib. & - & - \\
\hline Campanula latifolia $\mathrm{L}$. & Per. & $\mathrm{H}$ & Euro-Sib. & - & - \\
\hline Campanula olympica Boiss. & Per. & $\mathrm{H}$ & Euro-Sib. & - & - \\
\hline Compconula persicifolia $\mathrm{L}$. & Per. & $\mathrm{H}$ & Euro-Sib. & - & - \\
\hline Campanula rapunculoides $\mathrm{L}$. subsp. rapunculoides $\mathrm{L}$. & Per. & $\mathrm{H}$ & Euro-Sib. & - & - \\
\hline Campanula sibirica L. subsp. hohenackeri (Fisch. Et Mey.) Damboldt. & Per. & $\mathrm{H}$ & Euro-Sib. & - & - \\
\hline Legousia speculum-veneris (L.) Chaix. & Ann. & Th & Medit. & - & - \\
\hline \multicolumn{6}{|l|}{ Caprifoliaceae } \\
\hline Sambucus ebulus $\mathrm{L}$. & Per. & $\mathrm{H}$ & Euro-Sib. & - & - \\
\hline Sambucus nigra L. & Per. & $\mathrm{Ch}$ & Euro-Sib. & - & - \\
\hline \multicolumn{6}{|l|}{ Caryophyllaceae } \\
\hline Cerastium glomeratum Thuill. & Ann. & Th & - $\quad$ & - & - \\
\hline Dianthus carmelitarum Reut. Ex Boiss. & Per. & $\mathrm{H}$ & Euro-Sib. & End. & $\mathrm{LR}(\mathrm{lc})$ \\
\hline Minuartia circassica (Albow) Woron. & Per. & $\mathrm{H}$ & Euro-Sib. & - & - \\
\hline Saponaria prostrata Willd. subsp. calvertii (Boiss.) Hedge. & Bia. Per. & $\mathrm{H}$ & - & - & - \\
\hline Silene compacta Fischer. & Bia. Per. & $\mathrm{H}$ & - & - & - \\
\hline Silene multifida (Adams) Rohrb. & Per. & $\mathrm{H}$ & - & - & - \\
\hline Silene saxatilis Sims. & Per. & $\mathrm{H}$ & - & - & - \\
\hline Silene vulgaris (Moench) Garcke var. vulgaris (Moench) Garcke. & Per. & $\mathrm{H}$ & - & - & - \\
\hline \multicolumn{6}{|l|}{ Chenop odiaceae } \\
\hline Chenopodium album $\mathrm{L}$. & Ann. & Th & - & - & - \\
\hline Chenopodium folisum (Moench) Aschers. & Ann. & Th & - & - & - \\
\hline \multicolumn{6}{|l|}{ Cistaceae } \\
\hline Helianthemum mummularium (L.) Miller subsp. tomentosum & Per. & $\mathrm{Ch}$ & - & - & - \\
\hline
\end{tabular}


Table 5: Continue

Family and taxa of plants

Length of life Life-form

Element region End.

IUCN

(Scop.) Schinz Et Thellung.

Convolvulaceae

Convolvulus arvensis $\mathrm{L}$.

Convolvulus lineatus $\mathrm{L}$.

Crassulaceae

Sedum album $\mathrm{L}$.

Sempervivum armenum Boiss. Et Huet. var. armenum Boiss. Et Huet.

Datiscaceae

Datisca cannabina L.

Dipsacaceae

Dipsacus lacinictus L.

Scabiosa columbaria L. subsp. ochrolenca (L.) Celak

var. ochrolenca (L.) Coulter.

Scabiosa crinita Kotschy Et Boiss.

Ericaceae

Rhododendron luteum Sweet.

Rhododendron ungernii Trautv.

Rhododendron ponticum L. subsp. ponticum var. heterophyllum Ansin.

Vaccinium arctostophylos $\mathrm{L}$.

Vaccinium myrtillus $\mathrm{L}$.

Euphorbiaceae

Euphorbia amygdaloides L. var. anygdaloides L.

Euphorbia helioscopia L.

Euphorbia lathyris L.

Euphorbia stricta L.

Mercurialis annua $\mathrm{L}$.

Fabaceae (Leguminosae)

Anthyllis vulnercria L. subsp. boisseri (Sag.) Bornm.

Argyrolobium biebersteinii Ball.

Astragalus densifolius Lam. subsp. densifolius

Astragalus fragrans Willd.

Astragalus pontic us Pall.

Colutea cilicica Boiss. Et Bal.

Coronilla varia L. subsp. varia $\mathrm{L}$.

Dorycnium graecum (L.) Ser.

Galega officinalis L.

Genista tinctoria L.

Lathyrus aureus (Stev.) Brandza.

Lathyrus cicera L.

Lathyrus pratensis $\mathrm{L}$.

Lathyrus rotundifolius Willd. subsp. miniatus (Bieb. Ex Stev.) Davis.

Per.

Per.

Per.

Per.

Per.

Bia

Per.

Per.

Per.

Per.

Per.

Per.

Per.

Per.

Ann.

Per.

Ann.

Ann.

Bia. Per

Per.

Per.

Per.

Per.

Per.

Per.

Per.

Per.

Per.

Ann.

Ann.

Per.

Per.

Per.

Ann.

Per.

Per.

Ann.

Ann.

Ann.

Per.

Ann.

Bia.

Bia.

Per.

Per.

Ann.

Ann.

Ann.

Per.

Per.

Per.

Ann.

Ann.Bia

Per.

Ann.Bia.

Per.

Per.

Per.
Euro-Sib

End.

LR(lc)

r.-Tur.

Euro-Sib.

Euro-Sib.

Euro-Sib.

Euro-Sib.

Euro-Sib.

Euro-Sib.

Euro-Sib

Ir.-Tur.

Ir.-Tur

-

Euro-Sib.

Euro-Sib.

Euro-Sib.

Euro-Sib.

Euro-Sib

Medit.

Euro-Sib
End

EN

Trifolium medium L.

Trifolium nigrescens Viv.

Trifolium ochroleucum Huds.

Trifolium pannonicum Jacq. subsp. elongatum (Willd.) Zoh

Trifolium pratense $\mathrm{L}$.

Trifolium repens $\mathrm{L}$. var. repens $\mathrm{L}$. 
Table 5: Continue

Family and taxa of plants

Trifolium resupinatum $\mathrm{L}$. var resupinatum $\mathrm{L}$.

Trifolium sintenisii Freyn.

Trifolium subterraneum $\mathrm{L}$.

Vicia balansae Boiss.

Vicia cracca L. subsp. caracca L.

Vicia sativa L. subsp. nigra (L.) Ehrh.

Vicia tetrasperma (L.) Schreb.

Gentianaceae

Centaurium erythrae $a$ Rafn subsp. erythrae $a$ Rafn.

Gentiana asclepiadea $\mathrm{L}$

Gentiana cruciata L.

Gentiana nivalis L.

Gentiana pyrenaica L.

Geraniaceae

Gercanium asphodeloides Burm. Fil. subsp. sintenisii (Freyn) Davis.

Geranium ibericum Cav. subsp. jubatum (Hand.-Mazz.) Davis.

Geranium psilostemon Ledeb.

Geranium purpureum Vill.

Geranium sanguineum L.

Geranium stepporum Davis.

Geranium sylvaticum $\mathrm{L}$.

Gercaium roberticnum L.

Guttiferae (Hypericaceae)

Hypericum lydium Boiss.

Hypericum orientale $\mathrm{L}$.

Hypericum perforatum $\mathrm{L}$.

Hypericum scabrum $\mathrm{L}$.

Lamiaceae (Labiatae)

Ajuga orientalis $\mathrm{L}$.

Ajuga reptans $\mathrm{L}$.

Ballota nigra L. subsp. anatolica P.H. Davis.

Calamintha nepeta (L.) Savi subsp. glandulosa (Req.) P.W. Ball.

Calamintha sylvatica Bromf. subsp. sylvatica Bromf.

Glechoma hederacea $\mathrm{L}$.

Galeobdolon luteum Hudson subsp. luteum Hudson.

Galeopsis ladonum L.

Lamium album $\mathrm{L}$.

Lamium amplexicaule $\mathrm{L}$.

Lamium garganicum L. subsp. reniforme (Montbert Et

Aucher Ex Bentham) R. Mill.

Lamium purpureum $\mathrm{L}$.

Morrubium astraconicum Jacq. subsp. astraconicum Jacq.

Melissa officinalis $\mathrm{L}$. var. officinalis $\mathrm{L}$.

Mentha aquatica $\mathrm{L}$.

Mentha longifolia (L.) Hudson subsp. logifolia (L.) Hudson.

Mentha pulagium $\mathrm{L}$.

Origanum vulgare L. subsp. gracile (C. Koch) Et Swaart.

Phlomis russeliana (Sims) Beanthan.

Prunella vulgaris $\mathrm{L}$.

Salvia forskahle $\mathrm{L}$.

Salvia glutinosa $\mathrm{L}$

Salvia verticillota $\mathrm{L}$. subsp. verticillata $\mathrm{L}$.

Satureja spicigera (C. Koch) Boiss.

Stachys iberica Bieb. subsp. iberica Bieb. var. iberica Bieb.

Stachys macrantha (C. Koch) Stearn.

Teucrium orientale $\mathrm{L}$. var. orientale $\mathrm{L}$.

Thymus praecox Opiz subsp. jonkoe (Celak) Jalas var. jonkce Opiz.

Linaceae

Linum flavum L. subsp. flavum L.

Linum hypericifolium Salibs.

Linum nodiflorum $\mathrm{L}$.

Lytraceae

Lythrum salicaria $\mathrm{L}$.

Malvaceae

Abutilon theophrastii Medik.

Alcea hohenackeri (Boiss. Et Huet) Boiss.

Lavatera punctata All.

\begin{tabular}{|c|c|c|c|c|}
\hline Length of life & Life-form & Element region & End. & IUCN \\
\hline Ann. & Th & - & - & - \\
\hline Ann. & Th & Euro-Sib. & - & - \\
\hline Ann. & Th & - & - & - \\
\hline Per. & $\mathrm{H}$ & Euro-Sib. & - & - \\
\hline Ann. & Th & Euro-Sib. & - & - \\
\hline Ann. & Th & - & - & - \\
\hline Ann. & Th & - & - & - \\
\hline Bia.Per. & $\mathrm{H}$ & Euro-Sib. & - & - \\
\hline Per. & $\mathrm{H}$ & Euro-Sib. & - & - \\
\hline Per. & $\mathrm{H}$ & Euro-Sib. & - & - \\
\hline Ann. & Th & - & - & - \\
\hline Per. & $\mathrm{H}$ & Euro-Sib. & - & - \\
\hline Per. & $\mathrm{H}$ & Euro-Sib. & End. & LR(lc) \\
\hline Per. & $\mathrm{H}$ & Euro-Sib. & End. & LR(lc) \\
\hline Per. & $\mathrm{H}$ & Euro-Sib. & - & - \\
\hline Per. & $\mathrm{H}$ & - & - & - \\
\hline Per. & $\mathrm{H}$ & Euro-Sib. & - & - \\
\hline Per. & $\mathrm{H}$ & Ir.-Tur. & - & - \\
\hline Per. & $\mathrm{H}$ & Euro-Sib. & - & - \\
\hline Per. & $\mathrm{H}$ & - & - & - \\
\hline Per. & $\mathrm{H}$ & - & - & - \\
\hline Per. & $\mathrm{H}$ & - & - & - \\
\hline Per. & $\mathrm{H}$ & Euro-Sib. & - & - \\
\hline Per. & $\mathrm{H}$ & Ir.-Tur. & - & - \\
\hline Per. & $\mathrm{H}$ & - & - & - \\
\hline Per. & $\mathrm{H}$ & Euro-Sib. & - & - \\
\hline Per. & $\mathrm{H}$ & Ir.-Tur. & End. & $\mathrm{LR}(\mathrm{lc})$ \\
\hline Per. & $\mathrm{H}$ & - & - & - \\
\hline Per. & $\mathrm{H}$ & Euro-Sib. & - & - \\
\hline Per. & G & Euro-Sib. & - & - \\
\hline Per. & $\mathrm{H}$ & Euro-Sib. & - & - \\
\hline Ann. & Th & Euro-Sib. & - & - \\
\hline Per. & $\mathrm{H}$ & Euro-Sib. & - & - \\
\hline Bia. & $\mathrm{H}$ & Euro-Sib. & - & - \\
\hline Per. & $\mathrm{H}$ & - & - & - \\
\hline Ann. & Th & Euro-Sib. & - & - \\
\hline Per. & $\mathrm{H}$ & - & - & - \\
\hline Per. & $\mathrm{H}$ & - & - & - \\
\hline Per. & $\mathrm{H}$ & - & - & - \\
\hline Per. & $\mathrm{H}$ & Euro-Sib. & - & - \\
\hline Per. & $\mathrm{H}$ & - & - & - \\
\hline Per. & $\mathrm{H}$ & Ir.-Tur. & - & - \\
\hline Per. & $\mathrm{H}$ & Euro-Sib. & End. & $\mathrm{LR}(\mathrm{lc})$ \\
\hline Per. & $\mathrm{H}$ & Euro-Sib. & - & - \\
\hline Per. & $\mathrm{H}$ & Euro-Sib. & - & - \\
\hline Per. & $\mathrm{H}$ & Euro-Sib. & - & - \\
\hline Per. & $\mathrm{H}$ & Euro-Sib. & - & - \\
\hline Per. & $\mathrm{Ch}$ & Euro-Sib. & - & - \\
\hline Per. & $\mathrm{Ch}$ & Ir.-Tur. & - & - \\
\hline Per. & $\mathrm{H}$ & Euro-Sib. & - & - \\
\hline Per. & $\mathrm{H}$ & Ir.-Tur. & - & - \\
\hline Per. & $\mathrm{Ch}$ & - & - & - \\
\hline Per. & $\mathrm{H}$ & Euro-Sib. & - & - \\
\hline Per. & $\mathrm{H}$ & - & - & - \\
\hline Ann. & Th & Medit. & - & - \\
\hline Per. & $\mathrm{H}$ & Euro-Sib. & - & - \\
\hline Ann. & Th & - & - & - \\
\hline Per. & $\mathrm{H}$ & - & - & - \\
\hline Ann. & Th & - & - & - \\
\hline
\end{tabular}


Table 5: Continue

\begin{tabular}{|c|c|c|c|c|c|}
\hline Family and taxa of plants & Length of life & Life-form & Element region & End. & IUCN \\
\hline Malva neglecta Wallr. & Per. & $\mathrm{H}$ & - $\quad$ & - & - \\
\hline \multicolumn{6}{|l|}{ Onagraceae } \\
\hline Circae a lutetiona $\mathrm{L}$. & Per. & $\mathrm{H}$ & - & - & - \\
\hline \multicolumn{6}{|l|}{ (Hausskn.) P.H. Raven. } \\
\hline Epilobium angustifolium $\mathrm{L}$. & Per. & $\mathrm{H}$ & - & - & - \\
\hline Epilobium montanum L. & Per. & $\mathrm{H}$ & Euro-Sib. & - & - \\
\hline \multirow{2}{*}{\multicolumn{6}{|c|}{ Oxalidaceae }} \\
\hline & & & & & \\
\hline \multicolumn{6}{|l|}{ Papaveraceae } \\
\hline Fumaria officinalis $\mathrm{L}$. & Ann. & Th & - & - & - \\
\hline Popaver rhoeas $\mathrm{L}$. & Ann. & Th & - & - & - \\
\hline \multicolumn{6}{|l|}{ Parnassiaceae } \\
\hline Parnassia palustris $\mathrm{L}$. & Per. & $\mathrm{H}$ & - & - & - \\
\hline \multicolumn{6}{|l|}{ Plantaginaceae } \\
\hline Plantogo lenceolata $\mathrm{L}$. & Per. & $\mathrm{H}$ & - & - & - \\
\hline \multirow{2}{*}{\multicolumn{6}{|c|}{ Plumbaginaceae }} \\
\hline & & & & & \\
\hline \multicolumn{6}{|l|}{ Polygalaceae } \\
\hline \multicolumn{6}{|l|}{ Polygonaceae } \\
\hline Polygonum aviculare $\mathrm{L}$. & Ann. & Th & - & - & - \\
\hline Polygonum bistorta L. subsp. carneum (Koch) Coode Et Cullen. & Per. & $\mathrm{H}$ & Euro-Sib. & & \\
\hline Polygonum lapathifolium $\mathrm{L}$. & Ann. & Th & - $\quad$ & - & - \\
\hline Polygonum persicaria $\mathrm{L}$. & Ann. & Th & - & - & - \\
\hline Rumex acetosella $\mathrm{L}$. & Per. & $\mathrm{H}$ & - & - & - \\
\hline Rumex pulcher $\mathrm{L}$. & Per. & $\mathrm{H}$ & - & - & - \\
\hline Rumex crispus $\mathrm{L}$. & Per. & $\mathrm{G}$ & - & - & - \\
\hline \multicolumn{6}{|l|}{ Primulaceae } \\
\hline Anagalis arvensis $\mathrm{L}$. & Ann. & Th & - & - & - \\
\hline Cyclamen coum Miller var. coum Miller. & Per. & $\mathrm{G}$ & - & - & - \\
\hline Lysimachia verticillaris Srengel. & Per. & Hh & Euro-Sib. & - & - \\
\hline Primula auriculcta Lam. & Per. & $\mathrm{H}$ & Ir.-Tur. & - & - \\
\hline Primula elatior (L.) Hill subsp. meyeri (Rupr.) Valentine Et Lamond. & Per. & $\mathrm{H}$ & Euro-Sib. & - & - \\
\hline Primula elatior (L.) Hill subsp. pallasii (Lehm.) W.W. Sm. Et Forrest. & Per. & $\mathrm{H}$ & Euro-Sib. & - & - \\
\hline Primula veris L. subsp. columnae (Ten.) Ludi. & Per. & $\mathrm{H}$ & Euro-Sib. & - & - \\
\hline \multicolumn{6}{|l|}{ Ranunculaceae } \\
\hline Anemone narcissiflora L. subsp. narcissifflora L. & Per. & $\mathrm{H}$ & Euro-Sib. & - & - \\
\hline Caltha polypetala Hochst. Ex Lorent. & Per. & $\mathrm{H}$ & - $\quad$ & - & - \\
\hline Helleborus orientalis Lam. & Per. & $\mathrm{H}$ & Euro-Sib. & - & - \\
\hline Ranunc ulus arvensis $\mathrm{L}$. & Ann. & Th & - $\quad$ & - & - \\
\hline Ranunculus constantinopolitanus (DC.) D'Urv. & Per. & $\mathrm{H}$ & - & - & - \\
\hline Ranunc ulus kochii Ledeb. & Per. & $\mathrm{H}$ & Ir.-Tur. & - & - \\
\hline \multicolumn{6}{|l|}{ Rosaceae } \\
\hline Agrimonia eupatoria L. & Per. & $\mathrm{H}$ & - & - & - \\
\hline Alchemilla orduensis B. Pawl. & Per. & $\mathrm{H}$ & Euro-Sib. & End. & $\mathrm{EN}$ \\
\hline Alchemilla pseudocartalinica Juz. & Per. & $\mathrm{H}$ & - & - & - \\
\hline Alchemilla sintenisii Rothm. & Per. & $\mathrm{H}$ & Euro-Sib. & End. & $\mathrm{LR}(\mathrm{nt})$ \\
\hline Aremonia agrimonoides (L.) DC. & Per. & $\mathrm{H}$ & Euro-Sib. & - & - \\
\hline Cerasus avium (L.) Moench & Per. & $\mathrm{Ph}$ & - & - & - \\
\hline Filipendula vulgaris Moench. & Per. & $\mathrm{H}$ & Euro-Sib. & - & - \\
\hline Fragaria vesca $\mathrm{L}$ & Per. & $\mathrm{H}$ & - & - & - \\
\hline Geum coccineum $\mathrm{Sm}$. & Per. & $\mathrm{H}$ & Euro-Sib. & - & - \\
\hline Geum urbanum $\mathrm{L}$. & Per. & $\mathrm{H}$ & Euro-Sib. & - & - \\
\hline Mespilus germanica $\mathrm{L}$. & Per. & $\mathrm{Ph}$ & Euro-Sib. & - & - \\
\hline Potentilla crantzii (Crantz) G. Beck Ex Fritsch var. crantzii (Crantz) & Per. & $\mathrm{H}$ & Euro-Sib. & - & - \\
\hline \multicolumn{6}{|l|}{ G. Beck Ex Fritsch. } \\
\hline Potentillaerecta (L.) Rauschel. & Per. & $\mathrm{H}$ & - $\quad$ & - & - \\
\hline Potentilla humifisa Willd. & Per. & $\mathrm{H}$ & Euro-Sib. & - & - \\
\hline Potentilla micrantha Ramond Ex Dc. & Per. & $\mathrm{H}$ & - & - & - \\
\hline Rosa gallica L. & Per. & $\mathrm{Ch}$ & - & - & - \\
\hline Rosa canina $\mathrm{L}$ & Per. & $\mathrm{Ch}$ & - & - & - \\
\hline Rubus hirtus Waldst. Et Kit. & Per. & $\mathrm{Ch}$ & Euro-Sib. & - & - \\
\hline Rubus idaeus $\mathrm{L}$. & Per & $\mathrm{Ch}$ & - & - & - \\
\hline
\end{tabular}


Table 5: Continue

\begin{tabular}{|c|c|c|c|c|c|}
\hline Family and taxa of plants & Length of life & Life-form & Element region & End. & IUCN \\
\hline Rubus discolor Weihe Et Nees. & Per. & Ch & - & - & - \\
\hline Sanguisorba minor Scop. subsp. muricata (Spach) Briq. & Per. & $\mathrm{H}$ & - & - & - \\
\hline Sorbus aucuparia $\mathrm{L}$. & Per. & $\mathrm{Ph}$ & Euro-Sib. & - & - \\
\hline Sibbaldia parviflora Willd. var. parviflora Willd. & Per. & $\mathrm{Ch}$ & - & - & - \\
\hline \multicolumn{6}{|l|}{ Rubiaceae } \\
\hline Asperula pontica Boiss. & Per. & $\mathrm{H}$ & Euro-Sib. & - & - \\
\hline Asperula taurina L. subsp. caucasica (Poped.) Ehrend. & Per. & $\mathrm{H}$ & Euro-Sib. & - & - \\
\hline Asperula woronowii Krecz. & Per. & Ch & Euro-Sib. & End. & vU \\
\hline Cruciata taurica (Pallas Ex Willd.) Ehrend. & Per. & $\mathrm{H}$ & Ir.-Tur. & - & - \\
\hline Galium aparine $\mathrm{L}$. & Per. & $\mathrm{H}$ & - & - & - \\
\hline Galium verum L. subsp. verum $\mathrm{L}$. & Per. & $\mathrm{H}$ & Euro-Sib. & - & - \\
\hline \multicolumn{6}{|l|}{ Saxifragaceae } \\
\hline $\begin{array}{l}\text { Saxifraga cymbalaria } \mathrm{L} \text {. var. cymbalaria } \mathrm{L} \text {. } \\
\text { Scrophulariaceae }\end{array}$ & Ann. & Th & - & - & - \\
\hline \multicolumn{6}{|l|}{ Scrophulariaceae } \\
\hline $\begin{array}{l}\text { Digitalis ferruginea L. subsp. ferrugine a L. } \\
\text { Digitalis lomarckii Ivan. }\end{array}$ & Per. & $\mathrm{H}$ & Euro-Sib. & $-\bar{c}$ & - \\
\hline Euphrasia rostkoviana Hayne subsp. rostkoviana Hayne & Fei. & $\Pi$ & Furo-Sib. & Lilu. & - \\
\hline Kickxia elctine (L.) Dumort. & Ann. & Th & Medit. & - & - \\
\hline Linaria grandiflora Desf. & Per. & $\mathrm{H}$ & Ir.-Tur. & - & - \\
\hline Melampyrum arvense L. var. arvense $\mathrm{L}$. & Ann. & Th & Euro-Sib. & - & - \\
\hline Melampyrum arvense L. var. elatius Boiss. & Ann. & Th & Euro-Sib. & - & - \\
\hline Pedicularis comosa L. var. sibthorpii (Boiss.) Boiss. & Per. & $\mathrm{H}$ & - & - & - \\
\hline Pedicularis condensata Bieb. & Per. & $\mathrm{H}$ & Euro-Sib. & - & - \\
\hline $\begin{array}{l}\text { Rhinanthus angustifolius C.C. Gmelin subsp. grandiflorus } \\
\text { (Wallr.) D. A. Webb. }\end{array}$ & Ann. & Th & - & - & - \\
\hline Rhynchocorys stricta (C. Koch) Albov. & Ann. & Th & Euro-Sib. & - & - \\
\hline Scrophularia scopolï [Hoppe Ex] Pers. var. adenocalyx Somm. Et Lev. & Per. & $\mathrm{H}$ & Euro-Sib. & - & $\mathrm{CR}$ \\
\hline Verbascum gnophalodes $\mathrm{L}$. & Bia. & $\mathrm{H}$ & Euro-Sib. & - & - \\
\hline Verbascum phlomoides $\mathrm{L}$. & Bia. & $\mathrm{H}$ & Euro-Sib. & - & - \\
\hline Verbascum pyramidatum $\mathrm{L}$. & Bia. & $\mathrm{H}$ & Euro-Sib. & - & - \\
\hline Verbascum spectabile Bieb. var. spectabile Bieb. & Bia. & $\mathrm{H}$ & Euro-Sib. & - & - \\
\hline Verbascum thapsus $\mathrm{L}$. & Bia & $\mathrm{H}$ & Euro-Sib. & - & - \\
\hline Veronica anagallis-aquatica L. subsp. anagallis-aquatica $\mathrm{L}$. & Per. & $\mathrm{H}$ & - & - & - \\
\hline Veronica beccabunga L. subsp. beccabung $a \mathrm{~L}$. & Per. & $\mathrm{G}$ & - & - & - \\
\hline Veronica chamae drys $\mathrm{L}$. & Per. & $\mathrm{G}$ & Euro-Sib. & - & - \\
\hline Veronica cymbalaria Bodard. & Ann. & Th & Medit. & - & - \\
\hline Veronica filiformis J.E. Smith. & Per. & $\mathrm{H}$ & Euro-Sib. & - & - \\
\hline Veronica persica Poiret. & Ann. & Th & - $\quad$ & - & - \\
\hline \multicolumn{6}{|l|}{ Solanaceae } \\
\hline Atropa belladonna $\mathrm{L}$. & Per. & $\mathrm{H}$ & Euro-Sib. & - & - \\
\hline Datura stramonium $\mathrm{L}$. & Ann. & Th & - & - & - \\
\hline Hyocyamus niger L. & Ann. & Th & - & - & - \\
\hline Solomum dulcamara $\mathrm{L}$. & Ann. & Th & Euro-Sib. & - & - \\
\hline Solonum nigrum L. subsp. nigrum $\mathrm{L}$. & Ann. & Th & - & - & - \\
\hline \multicolumn{6}{|l|}{ Tamaricaeae } \\
\hline Myricaria germanica (L.) Desv. & Per. & $\mathrm{Ch}$ & - & - & - \\
\hline \multicolumn{6}{|l|}{ Thymelaeaceae } \\
\hline \multicolumn{6}{|l|}{ Urticaceae } \\
\hline Porietoria judaica L. & Per. & $\mathrm{H}$ & - & - & - \\
\hline Urtica dioica $\mathrm{L}$. & Per. & $\mathrm{H}$ & Euro-Sib. & - & - \\
\hline Urtica urens $\mathrm{L}$. & Per. & $\mathrm{H}$ & - & - & - \\
\hline \multicolumn{6}{|l|}{ Valerianaceae } \\
\hline Centranthus longiflorus Stev. subsp. longiflorus Stev. & Per. & $\mathrm{H}$ & Ir.-Tur. & - & - \\
\hline \multirow{2}{*}{\multicolumn{6}{|c|}{ Verbenaceae }} \\
\hline & & & & & \\
\hline Verbena officinalis $\mathrm{L}$. & Per. & $\mathrm{H}$ & - & - & - \\
\hline \multicolumn{6}{|l|}{ Violaceae } \\
\hline Viola alba Besser subsp. dehnhardtii (Ten.) Becker. & Per. & $\mathrm{H}$ & - & - & - \\
\hline Viola sieheana Becker. & Per. & $\mathrm{H}$ & - & - & - \\
\hline Viola tricolor $\mathrm{L}$. & Per. & $\mathrm{H}$ & - & - & - \\
\hline \multicolumn{6}{|l|}{ Monocotyledones } \\
\hline \multicolumn{6}{|l|}{ Alismataceae } \\
\hline Alisma lanceolatum with. & Per. & Hh & - & - & - \\
\hline Alisma plantago-aquatica $\mathrm{L}$. & Per. & Hh & Euro-Sib. & - & - \\
\hline
\end{tabular}


Table 5: Continue

\begin{tabular}{|c|c|c|c|c|c|}
\hline Family and taxa of plants & Length of life & Life-form & Element region & End. & IUCN \\
\hline \multicolumn{6}{|l|}{ Amaryllidaceae } \\
\hline Galanthus rizehensis Stern. & Per. & $\mathrm{G}$ & Euro-Sib. & - & vu \\
\hline Leucojum aestivum $\mathrm{L}$. & Per. & $\mathrm{G}$ & Euro-Sib. & - & - \\
\hline \multicolumn{6}{|l|}{ Araceae } \\
\hline Arum italicum Miller. & Per. & $\mathrm{G}$ & - & - & - \\
\hline Arum maculatum L. & Per. & $\mathrm{G}$ & - & - & - \\
\hline \multicolumn{6}{|l|}{ Cyperaceae } \\
\hline Carex echinata Murray. & Per. & $\mathrm{H}$ & Euro-Sib. & - & - \\
\hline Carex capitellata Boiss. Et Bal. & Per. & $\mathrm{H}$ & Euro-Sib. & - & - \\
\hline Carex ovalis Good. & Per. & $\mathrm{H}$ & Euro-Sib. & - & - \\
\hline Carex pallascens $\mathrm{L}$. var. chalcodeta (V. Krecz.) O. Nilsson. & Per. & $\mathrm{H}$ & Euro-Sib. & - & - \\
\hline Carex vesicaria $\mathrm{L}$. & Per. & $\mathrm{H}$ & - $\quad$ & - & - \\
\hline \multicolumn{6}{|l|}{ Iridaceae } \\
\hline Crocus kotschyomus C. Koch. subsp. suworowianus (C. Koch) Mathew. & Per. & $\mathrm{G}$ & - & - & - \\
\hline Gladiolus atroviolaceus Boiss. & Per. & $\mathrm{G}$ & Ir.-Tur. & - & - \\
\hline \multicolumn{6}{|l|}{ Juncaceae } \\
\hline Juncus articulatus $\mathrm{L}$. & Per. & Hh & Euro-Sib. & - & - \\
\hline Juncus inflexus $\mathrm{L}$. & Per. & Hh & - $\quad$ & - & - \\
\hline Luzula sylvatica (Hudson) Gaudin & Per. & $\mathrm{H}$ & Euro-Sib. & - & - \\
\hline \multicolumn{6}{|l|}{ Liliaceae } \\
\hline Allium olympicum Boiss. & Per. & $\mathrm{G}$ & Euro-Sib. & End. & - \\
\hline Allium scorodoprasum L. subsp. rotundum (L.) Stearn & Per. & $\mathrm{G}$ & Euro-Sib. & - & - \\
\hline Colchicum speciosum Steven & Per. & $\mathrm{G}$ & Euro-Sib. & - & - \\
\hline Gagea fistulosa Ker-Gawler. & Per. & $\mathrm{G}$ & - $\quad$ & - & - \\
\hline Muscari neglectum Guss. & Per. & $\mathrm{G}$ & - & - & - \\
\hline Muscari tenuiflorum Tausch. & Per. & G & - $\quad$ & - & - \\
\hline Lilium akkusianum R. Gamperle. & Per. & $\mathrm{G}$ & Euro-Sib. & End. & - \\
\hline Lilium ciliatum P.H. Davis & Per. & $\mathrm{G}$ & Euro-Sib. & End. & - \\
\hline Lilium monadelphum Bieb. var. armenum (Miscz. Ex & Per. & $\mathrm{G}$ & Euro-Sib. & - & - \\
\hline \multicolumn{6}{|l|}{ Grossh.) Davis Et Henderson } \\
\hline Ornithogalum oligophyllum E.D. Clarke. & Per. & $\mathrm{G}$ & - & - & - \\
\hline Ornithogalum orthophyllum Ten. & Per. & $\mathrm{G}$ & - & - & - \\
\hline Ornithogalum sigmoideum Freyn Et Sint. & Per. & $\mathrm{G}$ & Euro-Sib. & - & - \\
\hline Smilax excelsa L. & Per. & $\mathrm{Ch}$ & Medit. & - & - \\
\hline Veratrum album & Per. & G & Euro-Sib. & - & - \\
\hline \multicolumn{6}{|l|}{ Orchidaceae } \\
\hline Anacamptis pyramidalis (L) L.C.M. Richard. & Per. & $\mathrm{G}$ & - & - & - \\
\hline Dactylorhiza ilgazica Kreutz. & Per. & $\mathrm{G}$ & - & End. & - \\
\hline Dactylorhiza nieschalkiorum $\mathrm{H}$. Baumann Et Kunkele. & Per. & G & - & End. & - \\
\hline Dactylorhiza romana (Seb.) Soo. subsp. georgica (Klinge) Soo. & Per. & $\mathrm{G}$ & Euro-Sib. & - & - \\
\hline Dactylorhiza saccifera (Brongn.) Soo. & Per. & $\mathrm{G}$ & Medit. & - & - \\
\hline Dactylorhiza urville ana (Steudel) Baumann Et Kunkele. & Per. & $\mathrm{G}$ & Euro-Sib. & - & - \\
\hline Ophrys apifera Hudson & Per. & $\mathrm{G}$ & - & - & - \\
\hline Ophrys oestrifera Bieb. subsp. oestrifera Bieb. & Per. & $\mathrm{G}$ & Euro-Sib. & - & - \\
\hline Epipactis helloborne (L.) Crantz subsp. bithynica (Robartsch) Kreutz. & Per. & $\mathrm{G}$ & - & - & - \\
\hline Orchis provincialis Balbis Ex Dc. & Per. & $\mathrm{G}$ & Medit. & - & - \\
\hline Orchis purpurea Hudson. & Per. & $\mathrm{G}$ & Euro-Sib. & - & - \\
\hline Seropias feldwegiona $\mathrm{H}$. Baumann Et Kunkele. & Per. & G & Euro-Sib. & - & - \\
\hline Sercpias vomeracea (Burm. Fil.) Briq. subsp. orientalis Greuter. & Per. & $\mathrm{G}$ & Medit. & - & - \\
\hline \multicolumn{6}{|l|}{ Poaceae (Gramineae) } \\
\hline Agrostis gigantea Roth. & Per. & $\mathrm{H}$ & Euro-Sib. & - & - \\
\hline Agrostis stolonifera L. & Per. & $\mathrm{H}$ & Euro-Sib. & - & - \\
\hline Alopecurus arundinaceus Poiret. & Per. & $\mathrm{H}$ & Euro-Sib. & - & - \\
\hline Alopecurus myosuroides Hudson var. myosuroides Hudson. & Per. & $\mathrm{H}$ & Euro-Sib. & End. & - \\
\hline Arrhenatherum elatius (L.) & Per. & $\mathrm{H}$ & Euro-Sib. & - & - \\
\hline Bothriochloa ischaemum (L.) Keng. & Per. & $\mathrm{H}$ & - & - & - \\
\hline Brachypodium sylvaticum (Hudson) P. Beauv. & Per. & $\mathrm{H}$ & Euro-Sib. & - & - \\
\hline Brachypodium pinnatum (L.) P. Beauv. & Per. & $\mathrm{H}$ & Euro-Sib. & - & - \\
\hline Briza maxima $\mathrm{L}$. & Ann. & Th & - & - & - \\
\hline Bromus diandrus Roth. & Ann. & Th & - & - & - \\
\hline Bromus joponicus Thunb. subsp. japonicus Thunb. & Ann. & Th & - & - & - \\
\hline Bromus hordeaceus $\mathrm{L}$. & Ann. & Th & - & - & - \\
\hline Bromus sequarrosus L. & Ann. & Th & - & - & - \\
\hline Catabrosa aquatica (L) P. Beauv. & Per. & $\mathrm{H}$ & - & - & - \\
\hline Cynodon dactylon $\mathrm{L}$. & Per. & $\mathrm{H}$ & - & - & - \\
\hline Cynosurus cristotus $\mathrm{L}$. & Per. & $\mathrm{H}$ & Euro-Sib. & - & - \\
\hline Cynosurus echinatus $\mathrm{L}$. & Per. & $\mathrm{H}$ & Medit. & - & - \\
\hline
\end{tabular}




\begin{tabular}{|c|c|c|c|c|c|}
\hline Family and taxa of plants & Length of life & Life-form & Element region & End. & IUCN \\
\hline Dactylis glomerata L. subsp. glomerata L. & Per. & $\mathrm{H}$ & Euro-Sib. & - & - \\
\hline Deschampsiaflexuosa (L.) Trin. & Per. & $\mathrm{H}$ & Euro-Sib. & - & - \\
\hline Elymus repens $\mathrm{L}$. & Per. & $\mathrm{H}$ & - & - & - \\
\hline Eragrostis minor Host & Ann. & Th & - & - & - \\
\hline Festuca gigantea (L.) Vill. & Per. & $\mathrm{H}$ & Euro-Sib. & - & - \\
\hline Festuc a heteropphylla Lam. & Per. & $\mathrm{H}$ & Euro-Sib. & - & - \\
\hline Festuca pratensis Hudson. & Per. & $\mathrm{H}$ & - & - & - \\
\hline Festuca valesiaca Schleicher Ex Gaudin & Per. & $\mathrm{H}$ & - & - & - \\
\hline Gastridium ventricosum (Gouan) Schinz Et Thell. & Ann. & Th & Medit. & - & - \\
\hline Helictotrichon argaeum (Boiss) Parsa & Per. & $\mathrm{H}$ & Ir.-Tur. & End. & - \\
\hline Holcus lanatus $\mathrm{L}$. & Per. & $\mathrm{H}$ & Euro-Sib. & - & - \\
\hline Hordeum murinum L. subsp. glancum (Steudel) Tzvelev. & Per. & $\mathrm{H}$ & - & - & - \\
\hline Lolium perenne $\mathrm{L}$. & Per. & $\mathrm{H}$ & Euro-Sib. & - & - \\
\hline Lolium rigidum Gaudin var. rigidum Gaudin. & Per. & $\mathrm{H}$ & - & - & - \\
\hline Nordus stricta $\mathrm{L}$. & Per. & $\mathrm{H}$ & Euro-Sib. & - & - \\
\hline Paspalum dilatatum Poiret. & Per. & $\mathrm{H}$ & - & - & - \\
\hline Poa alpina L. subsp. fallax F. Hermann. & Per. & $\mathrm{H}$ & - & - & - \\
\hline Poa annua $\mathrm{L}$. & Ann. & Th & - & - & - \\
\hline Poa bulbosa L. & Per. & $\mathrm{H}$ & - & - & - \\
\hline Poa pratensis $\mathrm{L}$. & Per. & $\mathrm{H}$ & - & - & - \\
\hline Poa trivialis $\mathrm{L}$ & Per. & $\mathrm{H}$ & - & - & - \\
\hline Rostraria cristata (L.) Tzvelev var. cristata (L.) Tzvelev. & Ann. & Th & - & - & - \\
\hline Sesleria phleoides Steven Ex Roemer Et Schultes. & Per. & $\mathrm{H}$ & - & - & - \\
\hline Se t ar i a viridis (L.) P. Beauv. & Ann. & Th & - & - & - \\
\hline $\begin{array}{l}\text { Taeniatherum coput-medusae (L.) Nevski subsp. } \\
\text { crinitum (Schreber) Melderis. }\end{array}$ & Ann. & Th & Ir.-Tur. & - & - \\
\hline Vulpia bromoides (L.) S.F. Gray & Ann. & Th & - & - & - \\
\hline
\end{tabular}

\section{CONCLUSION}

As a result of the study, rangelands region of Ordu was observed to have a considerably rich flora with 246 genera, 461 species and subspecies belongs to 61 different families. Important results were found in the present study which was conducted to draw attention to and improve understanding of the plant variety of rangelans in the Ordu province. The present study was carried out in specific areas that are representative of the region and emphasizes the importance of the rangelans region in terms of plant diversity and endemism. The endemism rate of $6.89 \%$ was considered low compared to the rate of $16 \%$ estimated for the region (Ansin et al., 2002). Since, the region is located in the Euro-Siberian floral region, a great majority of the endemics belong to the same element $(4.28 \%)$. The endemics identified in the region include the following: Allium olympicum Boiss., Alopecurus myosuroides Hudson var. myosuroides Hudson., Astragalus densifolius Lam. subsp. densifolius, Centaurea sessilis Willd., Dactylorhiza nieschalkiorum H. Baumann Et Kunkele, Dianthus carmelitarum Reut. Ex Boiss., Heracleum platytaenium Boiss., Lathyrus czeczottianus Bassler., Lilium akkusianum R. Gamperle., Origanum vulgare L. subsp. gracile (C. Koch) Et Swaart., Ptilostemon afer (Jacq.) Greuter subsp. eburneus Greuter., Sempervivum armenum Boiss. Et Huet. var. armenum Boiss. Et Huet., Taraxacum turcicum Van Soest. In addition, 18 plants were determined as being under threat according to IUCN criteria including: Alchemilla orduensis B. Pawl., Anthemis tinctoria L., Cirsium trachylepis Boiss., Galanthus rizehensis Stern., Genista tinctoria L., Myosotis lazica M. Podov., Rhododendron ponticum L. subsp. ponticum var. heterophyllum Ansin.

Due to the topographical structure of the Eastern Black sea region, settlements are scattered. Many road construction studies have been carried out in order to reach villages, towns and high plateaus. Due to the rugged terrain, road construction damages the natural structure. In the regions where the transhumance tradition is widespread, people start to move to high plateaus during spring in order to feed animals and the vegetation cover sustains greater damage when they move to the high plateaus earlier than the usual season.

\section{RECOMMENDATIONS}

For protection of endemic and endangered plant species and so prevent the destruction of rangelands which are considered as natural resources and heritages, there is a need for preventing the excessive and disorganized benefitting from the rangelands hence, animal should graze throughout the grazing season. Enabling the uniform distribution of animals in the rangelans. Grazing with the nummber of animals compatible with grazing capacity of the rangelands. Should be grazing with the animal species compatible with the rangelands are extremely impotant. 
Moreover, some plants (medical plants, spices, ornamental plants, fuel, food, animal feeds, etc.) are collected from their natural habitats. In this case, uncontrolled plant collection would damage many plant types, particularly rare endemic and non-endemic plants. The following precautions are necessary in order to maintain the ecosystem which is the result of processes taking many years, in order to benefit from natural resources without harming the natural structure.

In many suitable places in Ordu region, botanical gardens and arboretums should be established where regional plants may be grown and kept. Plants that are picked from their natural habitat for medicinal use, spices, ornamental, fuel, food and animal feed plants should be checked and tested and cultivation studies should be accelerated.

Any facilities constructed in the region where high plateau tourism is developed should be built without harming the natural environment. Moreover, the construction of concrete buildings should not be permitted.

In regions where the high plateau culture is widespread, in order not to harm meadow species, early and over-grazing should be prevented. The dates on which livestock are moved to and returned from the high plateaus should be determined according to the development of the vegetation cover.

\section{ACKNOWLEDGEMENTS}

Researchers would like to thank Prof. Dr. Hamdi Guray Kutbay (Biology Department of Faculty of Science and Arts of Ondokuz Mayis University) and Prof. Dr. Rahim Ansin (Doga Schools; www.dogacollege.com) for their help in the diagnosis of plant samples.

\section{NOMENCLATURE}

$\begin{array}{ll}\text { Ann. } & =\text { Annual } \\ \text { Bia. } & =\text { Biannual } \\ \mathrm{Ch} & =\text { Chamaephytes } \\ \mathrm{CR} & =\text { Critically Endangered } \\ \mathrm{EN} & =\text { Endangered } \\ \text { Euro-Sib. } & \text { Euro-Siberian Elements } \\ \mathrm{G} & =\text { Geophytes } \\ \mathrm{H} & =\text { Hemicryptophytes } \\ \mathrm{Hh} & =\text { Hydrophytes } \\ \mathrm{Ir} .-\mathrm{Tur} . & =\text { Irano-Turanian elements } \\ \mathrm{IUCN} & =\text { International Union for the Conservation of } \\ & \\ \mathrm{lc} & =\text { Lature and Natural Resources } \\ \mathrm{LR} & =\text { Lover Risk }\end{array}$

$\begin{array}{lll}\mathrm{m} & =\text { Meter } \\ \mathrm{mm} & =\text { Milimeter } \\ \text { Medit. } & \text { Mediterranean elements } \\ \text { Mul. or Unk. P. Reg. }= & \text { Multiregional or Unknown } \\ & =\text { Phytogeographical Region } \\ \mathrm{nt} & =\text { Perennial } \\ \mathrm{Per} & = & \text { Phanerophytes } \\ \mathrm{Ph} & = & \text { Therophytes } \\ \text { Th } & = & \text { Turkish Plants Data Service } \\ \text { TUBIVES } & = & \text { Vulnerable } \\ \text { VU } & \end{array}$

\section{REFERENCES}

Akman, Y., 1999. Climate and Bioclimate (Bioclimate Methods and Turkey's Climate). Kariyer Press, Ankara, Turkey, Pages: 350.

Altay, V., I.I. Ozyigit and C. Yarci, 2010. Urban flora and ecological characteristics of the Kartal district (Istanbul): A contribution to urban ecology in Turkey. Sci. Res. Essay, 5: 183-200.

Andic, C., 1985. Existing plant species, its life forms and blooming periods in Erzurum province natural meadow, plateau and pasture. Ataturk Univ. J. Agric. Fac., 16: 85-104.

Andic, C., 1977. A Research on investigation of meadow and pasture vegetations of region of Erzurum on ecological and phytosociological. Ph.D. Thesis, Erzurum University, Turkey.

Anonymous, 2008. Meadow and Pasture lands of Turkey. Ministry of Agriculture and Rural Affairs, Agricultural Production and Development General Directorate, Ankara.

Ansin, R., 1980. Floristic descriptions of flora of East Black Sea Region and principal vegetation types. Ph.D. Thesis, Faculty of Forestry, Black Sea Technical University, Trabzon.

Ansin, R., 1983. Flora regions of Turkey and the principal vegetation types spreading across the region. J. Black Sea Tech. Univ. Faculty For., 6: 318-339.

Ansin, R., Z.C. Ozkan and O. Eminagaoglu, 2002. Endemic taxa of east black sea region (Endemic taxa of east black sea region) Artvin: The booklet of II. Nat. Black Sea For. Congr., 2: 565-573.

Asci, OO., 2011b. Biodiversity in red clover (Trifolium pratense L.) collected from Turkey I: Morphoagronomic properties. Afr. J. Biotechnol., 10: 14073-14079.

Asci, OO., 2011a. Salt tolerance in red clover (Trifolium pratense L.) seedlings. Afr. J. Biotechnol., 10: 8774-8781. 
Babalik, A.A. and H. Fakir, 2010. Plant species diversity in Isparta region rangelands. J. Anim. Vet. Adv., 9: 2788-2796.

Baytop, T., 1997. Turkish Dictionary of Plant Names. Presidency of High Agency of Ataturk. The Turkish Language Society Press, Ankara, Turkey.

Davis, P.H., 1985. Flora of Turkey and East Aegean Islands. Vol. 1-9, Edinburg University Press, Edinburg, UK.

Deveci, M. and C. Andic, 1992. Investigation of ecological and phytosociological of the natural Meadow-pasture vegetations of region of Van, Yuzuncuyil University. J. Inst. Sci., 1: 147-174.

Deveci, M., 2012. An investigation on plant species diversity in Colchic province (Turkey). Afr. J. Agric. Res., 7: 820-843.

Ekim, T., M. Koyuncu, M. Vural, H. Duman, Z. Aytac and N. Adiguzel, 2000. Red Data Book of Turkish Plants. Bariscan Press, Ankara, Turkey, Pages: 246.

Ellenberg, H. and D. Mueller-Dombois, 1967. A key to raunkiaer plant life forms with revised subdivisions. Ber. Geobot Inst. ETH Stiftg Rubel Zurich, 37: 56-73.

Eminagaoglu, A. and R. Ansin, 2003. The flora of Hatila Valley National Park and its close environs (Artvin). Turk. J. Bot., 27: 1-27.

Eminagaoglu, O. and R. Ansin, 2004. Flora of the Karagolsahara national park (Artvin) and its Environs. Turk. J. Bot., 28: 557-590.

Erik, S. and B. Tarikahya, 2004. On the flora of Turkey. Kebikec Human Resour. Res. J., 17: 139-163.

Guner, A., N. Ozhatay, T. Ekim and K.C. Baoer, 2000. Flora of Turkey and the East Aegean Islands. Vol. 11, Edinburgh University Press, Edinburgh, UK., pp: 656.

IUCN, 2001. IUCN Red List Categories. Version 3.1, IUCN Species Survival Commission, IUCN Gland, Switzerland and Cambridge, UK.

Karakaya, H. and M. Kilinc, 1996. The flora of the subalpine and alpine region of the cambasi high plateau (Ordu) and its vicinity. Turk. J. Bot., 20: 65-74.
McKey, D., 1994. Legumes and Nitrogen: The Evolutionary Ecology of a Nitrogen-Demanding Lifestyle. In: Advances in Legume Systematics, The Nitrogen Factor, Sprent, J.I. and D. McKey (Eds.). Royal Botanic Gardens, Kew, UK., pp: 211-228.

NPNC, 2007. National Biodiversity Strategy and Action Plan. Ministry of Environment and Forest, General Directorate of National Parks and Nature Conservation, Ankara, pp: 176.

Ozhatay, N. and S. Kultur, 2006. Check-list of additional taxa to the supplement Flora of Turkey III. Turk. J. Bot., 30: 281-316.

Ozhatay, N., S. Kultur and S. Aslan, 2009. Check-list of additional taxa to the supplement flora of Turkey IV. Turk. J. Bot., 33: 191-226.

Palaba, S. and R. Ansin, 2006. Subalpine and alpine flora of altindere valley (Macka, Trabzon). Turk. J. Bot., 30: 381-398.

Raunkiaer, C., 1934. The Life Form of Plants and Statistical Plant Geography. Oxford University Press, London, UK., Pages: 632.

Rundel, P.W., 1989. Ecological Success in Relation to Plant Form and Function in The Woody Legumes. In: Advances in Legume Biology (Monographs in Systematic Botany), Stirton, C.H. and J.L. Zarucchi (Eds.). Vol. 29. Missouri Botanical Garden Press, USA., Pages: 377-398.

Severoglu, Z., V. Altay, I.I. Ozyigit, M. Keskin and M. Serin et al., 2011. Some ecological charasteristics and The Flora of Golcuk District and its environs (Kocaeli-Turkey). Sci. Res. Essay, 6: 847-857.

Uzun, A. and S. Terzioglu, 2008. Vascular flora of forest vegetation in altindere valley (Macka-Trabzon). Turk. J. Bot., 32: 135-153.

Yaltirak, F. and A. Efe, 1996. Systematics of Herbaceous Plants Textbook Print II. Istanbul University Publication, Turkey, pp: 45-51.

Zohary, M. and D. Heller, 1984. The Genus Trifolium. The Israel Academy of Sciences and Humanities, Jerusalem, Israel, Pages: 606. 